\title{
Kinetic Theory and Fluid Dynamics
}

\author{
Yoshio Sone \\ Kyoto 606-0026, Japan
}

In this series of talks, I will discuss the fluid-dynamic-type equations that is derived from the Boltzmann equation as its the asymptotic behavior for small mean free path.

The study of the relation of the two systems describing the behavior of a gas, the fluid-dynamic system and the Boltzmann system, has a long history and many works have been done. The Hilbert expansion and the Chapman-Enskog expansion are well-known among them. The behavior of a gas in the continuum limit, however, is not so simple as is widely discussed by superficial understanding of these solutions. The correct behavior has to be investigated by classifying the physical situations. The results are largely different depending on the situations. There is an important class of problems for which neither the Euler equations nor the Navier-Stokes give the correct answer. In these two expansions themselves, an initial- or boundaryvalue problem is not taken into account. We will discuss the fluid-dynamic-type equations together with the boundary conditions that describe the behavior of the gas in the continuum limit by appropriately classifying the physical situations and taking the boundary condition into account.

Here the result for the time-independent case is summarized. The time-dependent case will also be mentioned in the talk.

The velocity distribution function approaches a Maxwellian $f_{e}$, whose parameters depend on the position in the gas, in the continuum limit. The fluid-dynamictype equations that determine the macroscopic variables in the limit differ considerably depending on the character of the Maxwellian. The systems are classified by the size of $\left|f_{e}-f_{e 0}\right| / f_{e 0}$, where $f_{e 0}$ is the stationary Maxwellian with the representative density and temperature in the gas.

(1) $\left|f_{e}-f_{e 0}\right| / f_{e 0}=O(\mathrm{Kn})$ (Kn : Knudsen number, i.e., $\mathrm{Kn}=\ell / L ; \ell$ : the reference mean free path. $L$ : the reference length of the system) : S system (the incompressible Navier-Stokes set with the energy equation modified). (1a) $\left|f_{e}-f_{e 0}\right| / f_{e 0}=o(\mathrm{Kn})$ : Linear system (the Stokes set).

(2) $\left|f_{e}-f_{e 0}\right| / f_{e 0}=O(1)$ with $\left|\int \xi_{i} f_{e} \mathbf{d} \boldsymbol{\xi}\right| / \int\left|\xi_{i}\right| f_{e} \mathbf{d} \boldsymbol{\xi}=O(\mathrm{Kn})\left(\xi_{i}\right.$ : the molecular velocity) : SB system [the temperature $T$ and density $\rho$ in the continuum limit are determined together with the flow velocity $v_{i}$ of the first order of Kn amplified by $1 / \mathrm{Kn}$ (the ghost effect), and the thermal stress of the order of $(\mathrm{Kn})^{2}$ must be retained in the equations (non-Navier-Stokes effect). The thermal creep[1] in the boundary condition must be taken into account.

(3) $\left|f_{e}-f_{e 0}\right| / f_{e 0}=O(1)$ with $\left|\int \xi_{i} f_{e} \mathbf{d} \boldsymbol{\xi}\right| / \int\left|\xi_{i}\right| f_{e} \mathbf{d} \boldsymbol{\xi}=O(1):$ E+VB system (the Euler and viscous boundary-layer sets). E system (Euler set) in the case where the boundary is an interface of the gas and its condensed phase.

The fluid-dynamic systems are classified in terms of the macroscopic parameters that appear in the boundary condition. Let $T_{w}$ and $\delta T_{w}$ be, respectively, the characteristic values of the temperature and its variation of the boundary. Then, the fluid-dynamic systems mentioned above are classified with the nondimensional temperature variation $\delta T_{w} / T_{w}$ and Reynolds number Re as shown in Fig. 1. In the region $\mathrm{SB}$, the classical gas dynamics is inapplicable, that is, neither the Euler 


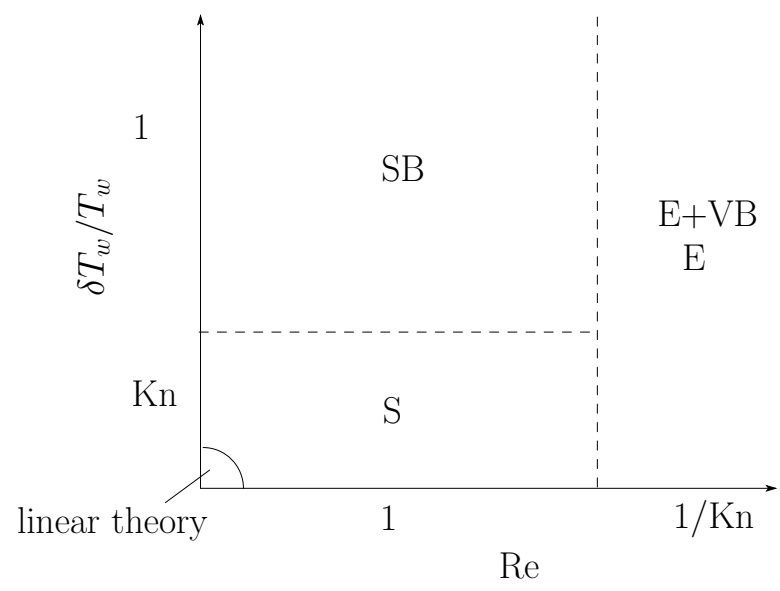

Figure 1: Fluid-dynamic-type systems describing the behavior of a gas of small Knudsen numbers $(\mathrm{Kn} \ll 1)$ in the parameter plane $\left(\mathrm{Re}, \delta T_{w} / T_{w}\right)$. In the region $\mathrm{SB}$, the classical gas dynamics is inapplicable.

equations nor the Navier-Stokes equation do not give the correct answer for the behavior of a gas in the continuum limit.

The above classification is done for general geometry. If a special geometry is considered, new non-Navier-Stokes and ghost effects, different from those mentioned above, enter the system, such as the stress quadratic of the shear of flow and the ghost effect of infinitesimal curvature of the boundary.[2]

\section{References}

[1] Y. Sone, Kinetic Theory and Fluid Dynamics (Birkhäuser, Boston, 2002).

[2] Y. Sone and T. Doi, "Ghost effect of infinitesimal curvature in the plane Couette flow of a gas in the continuum limit," Phys. Fluids 16, 952-971, (2004). 